\title{
Optimised simulated annealing for Ising spin glasses
}

\author{
S.V. Isakov ${ }^{1}$, I.N. Zintchenko, T.F. Rønnow, M. Troyer \\ Theoretische Physik, ETH Zurich, 8093 Zurich, Switzerland
}

\begin{abstract}
We present several efficient implementations of the simulated annealing algorithm for Ising spin glasses on sparse graphs. In particular, we provide a generic code for any choice of couplings, an optimised code for bipartite graphs, and highly optimised implementations using multi-spin coding for graphs with small maximum degree and discrete couplings with a finite range. The latter codes achieve up to 50 spin flips per nanosecond on modern Intel CPUs. We also compare the performance of the codes to that of the special purpose D-Wave devices built for solving such Ising spin glass problems.
\end{abstract}

Keywords: spin glasses, optimisation, simulated annealing

\section{PROGRAM SUMMARY}

Program Title: SimAn v1.0

Journal Reference:

Catalogue identifier:

Licensing provisions: GPLv3

Programming language: $\mathrm{C}++$, OpenMP for parallelization.

Computer: any PC.

Operating system: Linux/OS X/UNIX.

Has the code been vectorized or parallelized?: parallelized using OpenMP.

RAM: Variable, from a few megabytes.

Number of processors used: Variable.

Keywords: spin glasses, optimisation, simulated annealing.

Classification: $4.13,6.5,23$.

Nature of problem: Ising spin glass ground states on sparse graphs.

Solution method: Simulated annealing.

Running time: From milliseconds to seconds.

\section{Introduction}

First introduced three decades ago [1], simulated annealing is a powerful algorithm commonly used for heuristic optimisation due to its simplicity and effectiveness. Within this approach, variables to be optimised are viewed as the degrees of freedom of a physical system and the cost function of the optimisation problem as the energy. One then performs a Monte Carlo simulation of that system, starting at high temperatures and slowly lowering the temperature during the simulation, so that ultimately the configuration of the system ends up in a local minimum. Annealing slow enough and with multiple repetitions, one can hope to find the global minimum.

In this paper we present highly optimised implementations of simulated annealing for the Ising spin glass problem

$$
H=\sum_{i<j} J_{i j} s_{i} s_{j}+\sum_{i} h_{i} s_{i}
$$

\footnotetext{
${ }^{1}$ Current address: Google, Brandschenkestrasse 110, 8002 Zurich, Switzerland
} 
where $s_{i}= \pm 1$. The couplings $J_{i j}$ induce a graph structure with the spins represented as vertices and with edges between all neighbour pairs $i$ and $j$ for which $J_{i j} \neq 0$.

The broad interest in the Ising spin glass comes from the fact that finding the ground state is non-deterministic polynomial (NP) hard [2]. This means that many other interesting problems, including constraint satisfaction and the travelling salesman problem, can be mapped to such Ising spin glasses in polynomial time.

The complexity of solving this problem is also the motivation behind special purpose devices built by the company D-Wave systems, which can to date solve Ising spin glass problems with up to $N=512$ spins on the so-called "chimera graph" (discussed in Appendix A). The existence of these devices has triggered increased efforts into effectively mapping non-linear combinatorial optimisation problems from application domains, such as image recognition, to Ising spin glass problems [3, 4]. A recent study [5] has compared the performance of a D-Wave device against three general purpose classical optimisation algorithms and concluded that the D-Wave device tested was 3600 times faster than the fastest of these codes. In their conclusions, the authors qualify their result with the statement "It would of course be interesting to see if highly tuned implementations of simulated annealing could compete". The optimised simulated annealing codes for Ising spin glasses presented in this publication can also be applied to the chimera graph of the D-Wave devices and provide such competitive highly tuned implementations.

Another state-of-the-art approach for finding ground states of spin glasses is parallel tempering [6, 7]. This method can be more efficient than simulated annealing in some cases [8, 9], e.g. for Ising spin glasses with Gaussian distribution of couplings. Most of the optimisation techniques presented in this paper can also be applied to parallel tempering.

\section{Optimisations}

Simulated annealing is simple and can be implemented in a short time for the Ising spin glass. However, a range of optimisations can improve its performance by orders of magnitude. In this work we discuss many of these optimisations and present efficient implementations for modern CPUs in a freely available software package.

\subsection{Forward computation of $\Delta E$}

Performing simulated annealing using the Metropolis algorithm requires calculating the acceptance $\operatorname{ratio} \exp \left(-\beta \Delta E_{i}\right)$, where $\beta$ is the inverse temperature and $\Delta E_{i}$ the energy change upon flipping the $i$ 'th spin. The value of $\Delta E_{i}$ is typically computed by traversing the neighbours of spin $i$ and takes up most of the time required for each spin update. However, as for typical annealing schedules the average acceptance rate is only around $15 \%$, it is much more efficient to calculate and store $\Delta E_{i}$ for every spin and only update this value if spin $i$, or one of its neighbours, is flipped. This way the number of operations if a spin-flip is accepted is the same with an additional array access. On the other hand, if a flip is not accepted, $\Delta E_{i}$ does not have to be computed, but simply retrieved from an array.

\subsection{Fixed loop lengths and unrolling}

One has to loop over neighbours to compute the energy change $\Delta E_{i}$ when flipping the $i$-th spin. This loop can be optimised using fixed loop lengths by specifying the maximum number of neighbours at compile time. In this case, the compiler can unroll the loop more efficiently. This approach is advantageous when the distribution of the number of neighbours is narrow. For instance, for perfect chimera graphs with five and six neighbours (there might be a few sites with four neighbours in depleted graphs) the code with fixed loop length is $20 \%$ faster. However, using the fixed loop length codes might be disadvantageous when the distribution of the number of neighbours is wide, say, for graphs with the majority of sites having three neighbours and a few sites having ten neighbours.

\subsection{Fast random number generators}

For a simple model, like the Ising model, generation of random numbers can take up a substantial fraction of the computational effort. Unlike simulations aiming at high accuracy results for physical properties, in optimisation algorithms such as simulated annealing the quality of the random number generator is not very critical and thus fast generators, such as the linear-congruential, can be used. 


\subsection{Deterministic traversal over lattice sites}

Lattice sites can be picked up for an update sequentially in some specified order instead of picking them up in random order. This approach decreases the amount of generated random numbers and leads to faster codes. Even though the detailed balance condition is violated, it typically yields betters success rates.

\subsection{Precomputing random numbers}

Random numbers can also be reused across multiple repetitions of the annealing, as long as they start from different initial configurations. Furthermore, we can modify the Metropolis acceptance criterion from $\exp \left(-\beta \Delta E_{i}\right)<u$, where $u \in[0,1)$ is drawn uniformly at random, to a cheaper decision $\Delta E<r$, where $r=-\frac{1}{\beta} \log u$. The values $r$ can then be stored instead of $u$. For the case of integer couplings we can further optimise by using integer comparison instead of floating point comparisons.

Correlations introduced by reusing random numbers can be significantly reduced with minimal additional effort by cyclically shifting the precomputed array of random numbers for each sweep (a sweep is defined as one attempted update per spin) by a random offset. We observed that the remaining correlations have only a minimal impact on the performance of the simulated annealer.

\subsection{Optimisations for bipartite graphs}

If the graph is bipartite, the complexity of finding the ground state configuration can be reduced to finding the optimal spin-vector for only one sub-lattice. Lets split the set of spins into two sets $A$ and $B$ such that the spins from one set only couple to spins of the other. Without loss of generality we assume that all on-site fields $h_{i}=0, N_{A} \leq N_{B}$ and lets sort the spins such that all spins in $A$ come before those in $B$. We use the notation $\mathbf{s}_{A}=\left\{s_{1}, s_{2}, \ldots, s_{N_{A}}\right\}^{T}$, $\mathbf{s}_{B}=\left\{s_{N_{A}+1}, s_{N_{A}+2}, \ldots, s_{N}\right\}^{T}$ and $\mathbf{s}=\left\{\mathbf{s}_{A}, \mathbf{s}_{B}\right\}$. The couplings $J_{i j}$ are then in matrix form

$$
J=\left(\begin{array}{cc}
\mathbf{0} & C^{T} \\
C & \mathbf{0}
\end{array}\right)
$$

where the energy can be calculated as

$$
E=\frac{1}{2} \mathbf{s}^{T} J \mathbf{s}=\frac{1}{2}\left(\mathbf{s}_{A}^{T} C^{T} \mathbf{s}_{B}+\mathbf{s}_{B}^{T} C \mathbf{s}_{A}\right)=\mathbf{s}_{B}^{T} C \mathbf{s}_{A} .
$$

which can be minimised by finding the optimum $\mathbf{s}_{A}$ such that

$$
E=\min _{\mathbf{s}_{A}}\left\{-\sum_{i=0}^{N_{B}}\left|\sum_{j=0}^{N_{A}} C_{i j} s_{j}\right|\right\}
$$

as we can always align the spins in $\mathbf{s}_{B}$ accordingly. Therefore, for bipartite graphs we need to simulate and update only $N_{A} \leq N / 2$ spins. In the above argument we for simplicity made the assumption that all on-site fields are zero. If this is not the case, we can transform the Hamiltonian by introducing two ancillary spins, one coupled to all spins in sublattice $A$ with couplings $h_{i}$ for $i \in\left[1, N_{A}\right]$ and the other to all spins in sub-lattice $B$ with couplings $h_{i}$ for $i \in\left[N_{A}+1, N\right]$. The two spins are also coupled to each other with a strong ferromagnetic bond. After this transformation, the graph remains bipartite, but all on-site fields are expressed as couplings between spins and the above argument can be applied.

\subsection{Multi-spin coding}

In contrast to the standard implementations of simulated annealing where one uses an integer to store every spin, higher efficiency can be archived by representing spins by single bits which allows one to update many spins simultaneously. This approach is known as multi-spin coding. We here present two different implementations of multi-spin coded simulated annealers. The codes were written for different ranges of couplings and with up to six nearest neighbours, using words of $S=64$ bits to stores 64 spins. Rather than storing spins from a single lattice across a word, as described in [10], we store 64 replicas of the same spin in one word. 


\begin{tabular}{c|c|c|c} 
Index $i$ & Energy $E_{i}$ & Energy gain $\Delta E_{i}$ & Probability $p_{t, i}$ \\
\hline 0 & $-3|J|$ & $6|J|$ & $\exp (-6 \beta|J|)$ \\
1 & $-1|J|$ & $2|J|$ & $\exp (-2 \beta|J|)$ \\
2 & $1|J|$ & $-2|J|$ & 1 \\
3 & $3|J|$ & $-6|J|$ & 1
\end{tabular}

Table 1: Indices, energies, energy gains when a spin is flipped, and probabilities for one spin that couples to three neighbours with $|J|=1$.

\subsubsection{Approach one}

Multi-spin coded versions of simulated annealing were first suggested in Ref. [11] and later extended in Refs. [1214]. The implementation presented here is based on the outline found in Ref. [14]. For completeness, we summarise this approach. In a standard Monte Carlo simulation of the Ising model, one selects a random spin and computes the energy $\Delta E$ gained by flipping this spin. The move is either accepted or rejected with a probability given by the Boltzmann factor $e^{-\beta \Delta E}$, where $\beta$ is the inverse temperature. In the approach suggested in Ref. [14], this part of the algorithm is slightly altered. For a finite number of lattice neighbours and integer couplings, the number of all possible values of $\Delta E$ is finite and these values can be ordered in descending order $\Delta E \in\left\{-2 E_{0}, \ldots,-2 E_{m}\right\}$, where $E_{i}$ are the local energy levels of the spin and $m+1$ is the number of energy levels. Instead of calculating $\Delta E$, one can efficiently calculate the index $i$ of $-2 E_{i}$ for all the 64 spins simultaneously by bitwise summation, i.e. indices are stored in $\left\lceil\log _{2}(k)\right\rceil$ words, where \lceil\rceil denotes the next largest integer value. The spins which need to be flipped with probabilities $p_{t, i}=e^{2 \beta E_{i}}$ can be determined by performing simple boolean logic on words that represent indices $i$ and by comparing the probabilities $p_{t, i}$ with a uniformly distributed random number $0 \leq u<1$ starting at the highest through the lowest level. This can be illustrated by the following pseudo code example for a spin that couples to three neighbours with $J_{i j}= \pm 1$.

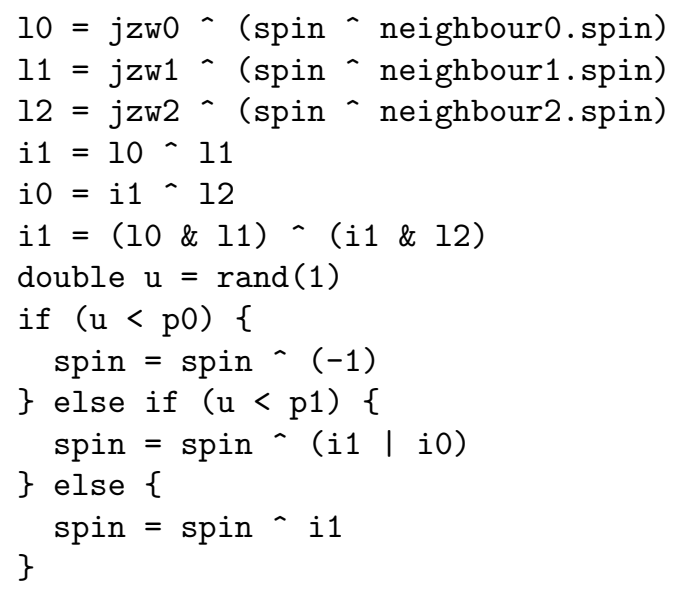

Here spin, jzw0, jzw1, jzw2, 10, 11, 12, i0, and i1 are 64-bit words, spin stores 64 spins for 64 replicas, jzw represents the coupling constant $J$ (all bits of jzw are set to zero if $J=1$ and all bits of jzw are set to one if $J=-1$ ). In the first three lines of the code, we determine whether the interaction energy is positive or negative for every pair of interacting spins. A bit of $l j$ is set to one if the corresponding interaction energy is positive and set to zero if the interaction energy is negative. In the next three lines of the code, we calculate the index $i$ by bitwise summation of 10, 11, and 12. In this simple example, we need only two words to store the index. All possible indices $i$ for one replica, corresponding energies $E_{i}$, energy gains $\Delta E_{i}$, and probabilities $p_{t, i}$ are listed in table 1 . In the seventh line, we draw a uniformly distributed random number. In the next lines, we compare it to the probabilities $p_{t, i}$ and flip spins. It is easy to deduce from the correspondence between indices and probabilities which spins should be flipped. Namely, we find by simple boolean logic the spins with indices $i \geq k$ such that $p_{k-1, t}<u<p_{k, t}$ and flip these spins. As the probabilities $p_{i, t}$ are used often throughout the simulations, these are precomputed when the algorithm is initialised.

Correlations. This approach results in correlations between the replicas because only one random number is used per update for all the 64 replicas in a word. For example, if at any point during the annealing two replicas are in the same 

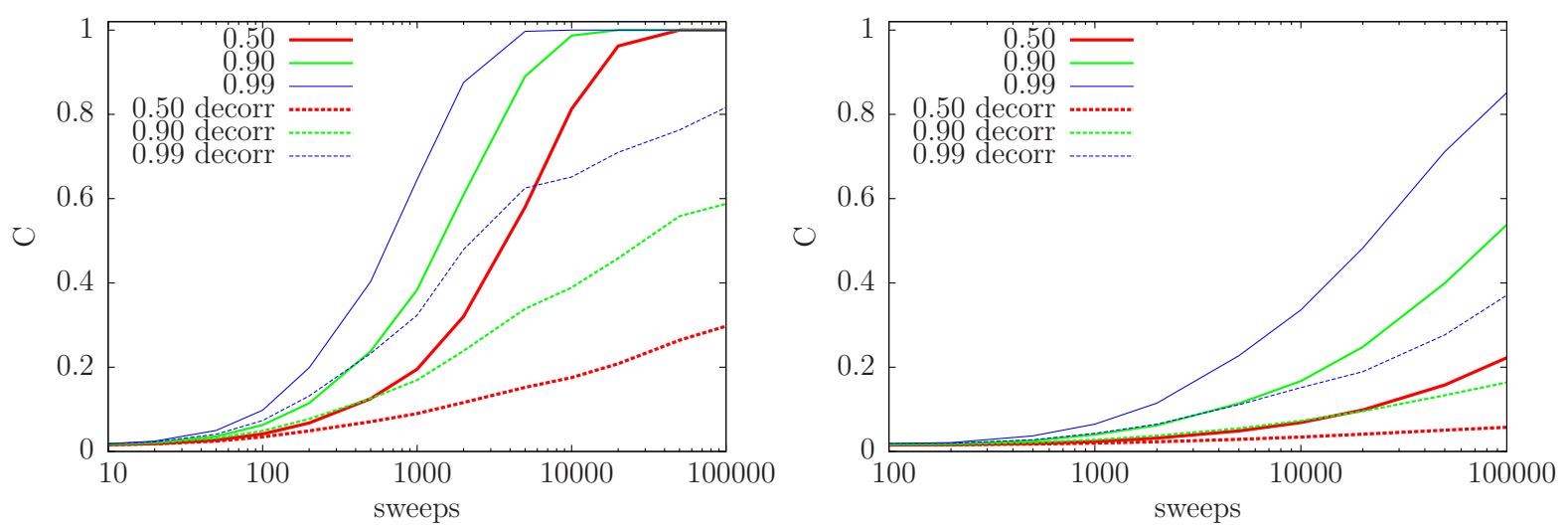

Figure 1: The correlation ratio $C$ as a function of the number of sweeps for instances without fields on depleted chimera graphs with 126 spins in the left panel and 503 spins in the right panel (see Appendix A for details). The data are presented for the 0.5, 0.9 and 0.99 quantiles. The definition of quantile is given at the beginning of Sec. 3.2. The dotted and solid curves show the correlations introduced by the code with and without applying the decorrelation strategy respectively.

state, they will follow the same path, making one of the replicas redundant. In the extreme case of fully correlated replicas, all of them find the same state. Correlations can be measured by computing the correlation ratio

$$
C=\frac{V}{W(1-W)}
$$

over multiple repetitions of the annealing process, where $W=(1 / R) \sum_{i=1}^{R} w_{i}$ is the mean success rate (the probability of finding the ground state), $R$ is the number of repetitions, $V=(1 / R) \sum_{i=1}^{R}\left(w_{i}-W\right)^{2}$ is the variance and $w_{i}=$ $(1 / 64) \sum_{j=1}^{64} w_{i j}$ is the mean success rate of the $i$ th repetition (each repetition has $S=64$ replicas). It can be shown that $C$ is close to zero for uncorrelated replicas and $C=1$ for fully correlated replicas.

In figure 1, we show the correlation ratio $C$ as a function of the number of sweeps for instances on the chimera graph. Correlations increase with the ratio of the number of sweeps to the system size. However, they can be substantially reduced by not flipping one random spin (bit) in each update. The random number that is used to make an update can be reused. As can be seen, our decorrelation strategy reduces the correlations significantly but not fully. It should be emphasised that these correlations are usually irrelevant because strong correlations appear only when the number of sweeps is much larger than the optimal number of sweeps to find the ground state with very high probability, as discussed below.

\subsubsection{Approach Two}

The second approach follows the ordinary algorithm where a spin is picked, its local energy is computed and it is flipped with probability $p$. However, instead of just flipping one spin, one determines the individual energies of 64 spins simultaneously and computes whether the spins should be flipped from a set of probabilities. The average case complexity of generating $Q 1$-bits with probability $p$ in parallel is $O\left(\log _{2}(Q)+2\right)$ [15]. This way 64 bits are generated in on average 8 iterations. Since we are considering sparse graphs with a limited range, only a limited number of flipping probabilities can be attained by a spin at each time step and this makes the generalisation of the above algorithm to individual flipping probabilities $p^{(1)}, p^{(2)}, \ldots, p^{(Q)}$ straight forward. While this algorithm is more than a factor of three slower than the one presented in Sec. 2.7.1, correlations are here of the order of the pseudo random number generator.

\section{Optimising annealing strategies}

It is important to optimise both the slope and the length of the annealing schedule. 


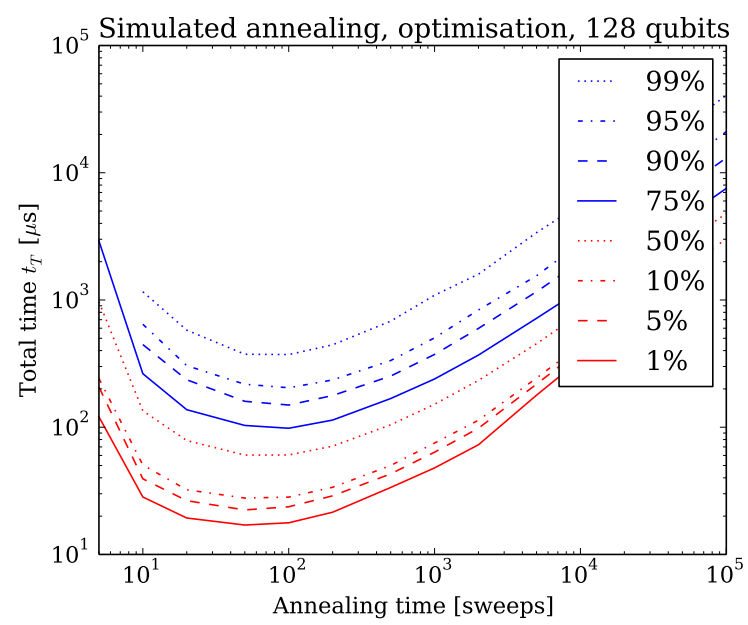

Figure 2: Quantile optimisation for $\pm J$ instances on the chimera graph.

\subsection{Optimising the schedules}

We follow the ideas in Ref. [16]. Based on considerations of keeping the average energy difference between two successive steps $k$ and $k+1$ below a threshold $\left\langle E_{k+1}\right\rangle-\left\langle E_{k}\right\rangle \leq-\lambda \sigma_{k}$ it can be shown that $\beta_{k+1}=\beta_{k}+\lambda \sigma_{k}^{-1}$ where $\sigma_{k}$ is the standard deviation of the energy at step $k$ and $\lambda \leq 1$. We optimised the schedule using this approach, but found that the performance was only slightly better than a linear schedule. Much more important is that the starting value of the temperature $T$ is around the same order as the maximal energy required to flip any spin and that the end value is low enough such that the state does not jump out of the final minimum. For bi-modal couplings $J_{i j}= \pm 1$, we found that the inverse temperatures $\beta_{s}=0.1$ and $\beta_{e}=3$ were good initial and final values up to 512 spin problems, and these values were used for the benchmark runs.

\subsection{Optimising annealing times}

As simulated annealing is a heuristic algorithm, one can strive towards maximising the probability of finding the ground state by either increasing the number of sweeps, increasing the number of repetitions, or both at once. The optimal choice which achieves this goal with minimal total computational effort depends on the the class of problem at hand. Furthermore, for a set of such problems, how should one run the code to lower the computational resources needed for 5\% easiest ones? For the 50\% easiest? Or for the $99 \%$-easiest? We refer to the various percentages as quantiles, and to address these questions, we consider an annealer which is ran using $S$ sweeps with $R$ repetitions. If the probability of finding the ground state for a single repetition is $w(S)$, then the total number of repetitions needed to find the ground state with $99 \%$ is

$$
R=\left\lceil\frac{\log (0.01)}{\log (1-w)}\right\rceil,
$$

where we take the ceiling \lceil\rceil as the number of repetitions of annealing must be an integer. This gives a total annealing time of

$$
t_{T}=t_{a} \cdot R
$$

Here $t_{a}$ is the annealing time for one repetition which is given by $t_{a}=S \cdot N / f$ (when $S \cdot N$ is large), where $S$ is the number of sweeps performed in the simulation, $N$ is the system size and $f$ is the number of attempted spin updates per second. Since $w\left(t_{a}\right)$ is a non-trivial function of $t_{a}$, the total time $t_{T}$ to find the ground state with $99 \%$ probability is a non-trivial function of $t_{a}$, and therefore one needs to minimise $t_{T}$ as a function of $t_{a}$ in order to find the optimal running parameters for the algorithm. As an example here consider 1000 random problems on the chimera graph (Appendix A). We then plot $t_{T}\left(t_{a}\right)$ in fig. 2. It is evident that the code runs optimally when ran between $t_{a}=400 \cdot N / f$ and $t_{a}=1000 \cdot N / f$ depending on which quantile the problem belongs to. 


\begin{tabular}{|c|c|}
\hline Code & Description \\
\hline an_ms_r1_nf & Multi-spin code for range-1 interactions without magnetic field (approach one). \\
\hline an_ms_r1_fi & Multi-spin code for range-1 interactions with magnetic field (approach one). \\
\hline an_ms_r3_nf & Multi-spin code for range-3 interactions without magnetic field (approach one). \\
\hline an_ms_r1_nf_v0 & Multi-spin code for range- 1 interactions without magnetic field (approach two). \\
\hline an_ss_ge_fi & Single-spin code for general interactions with magnetic field (fixed number of neighbours). \\
\hline an_ss_ge_fi_vdeg & Single-spin code for general interactions with magnetic field (any number of neighbours). \\
\hline an_ss_ge_nf_bp & $\begin{array}{l}\text { Single-spin code for general interactions on bipartite lattices without magnetic field } \\
\text { (fixed number of neighbours). }\end{array}$ \\
\hline an_ss_ge_nf_bp_vdeg & $\begin{array}{l}\text { Single-spin code for general interactions on bipartite lattices without magnetic field } \\
\text { (any number of neighbours). }\end{array}$ \\
\hline an_ss_ge_fi_bp_vdeg & $\begin{array}{l}\text { Single-spin code for general interactions on bipartite lattices with magnetic field } \\
\text { (any number of neighbours). }\end{array}$ \\
\hline an_ss_rn_fi & Single-spin code for range- $n$ interactions with magnetic field (fixed number of neighbours). \\
\hline an_ss_rn_fi_vdeg & Single-spin code for range- $n$ interactions with magnetic field (any number of neighbours). \\
\hline
\end{tabular}

Table 2: A list of simulated annealing codes. These are also targets (executable names) for the Makefile.

We also consider the mean time to find the ground state [5], which is given by

$$
t_{M}=t_{a}\left\lceil\frac{1}{w}\right\rceil .
$$

That is we replaced $\log (0.01) / \log (1-w)$ by $1 / w$ in eq. 6 . If $w$ is small then the mean time to find the ground state is a time to find the ground state with $63 \%$ probability.

\section{Simulated annealing codes}

We provide a number of simulated annealing codes. The codes are listed in table 2. Most of the multi-spin codes are designed for lattices that have from one to six neighbours at each site and with couplings that can be \pm 1 . The codes can be sped up by choosing the required number of neighbours in the ms_config.h file. For instance, one can leave just the \#define USE_4_NEIGHB line and comment out the other five lines for the square lattice.

We have also developed multi-spin codes for other interaction ranges (by range- $n$ we mean integer couplings within a range $[-n,-n+1, \ldots,-1,1, \ldots, n-1, n])$ and for lattices with larger (fixed) number of neighbours. The programming of higher range codes is rather tedious, so we use a code generator to generate these codes. We include range- 3 code for up to six neighbours. The other codes can be obtained from the authors.

There are two sets of single-spin codes. The codes that end with vdeg use variable loop lengths over neighbours and designed for any number of neighbours. The other codes use fixed loop lengths and the maximum number of neighbours can be set in the ss_config.h file (the default value is six). The latter set of codes can be faster in some circumstances as discussed in Sec. 2.2.

To find the ground state, annealing must be repeated many times as described is Sec. 3.2. The loop over repetitions can be easily parallelized using OpenMP as the repetitions are independent of each other. This can lead to a significant speedup (up to a factor of the number of threads used) when the number of repetitions is large enough. We provide both single-threaded and multi-threaded versions of the codes.

\subsection{Building the codes}

The codes are all stand-alone and can be built using a $\mathrm{C}++11$ conforming compiler as follows: make target, where target specifies the code to build. All available targets are listed in table 2. To build a multi-threaded version append_omp to target. 


\begin{tabular}{|c|c|}
\hline Argument flag & Description \\
\hline-1 [instance] & [instance] specifies instance file. \\
\hline$-s \quad[$ sweeps $]$ & [sweeps] is number of sweeps. \\
\hline$-r$ [reps] & [reps] is number of repetitions. \\
\hline -b0 [beta0] & [beta0] is initial inverse temperature. Default value: 0.1 . \\
\hline -b1 [beta1] & [beta1] is final inverse temperature. Default value: 3.0 . \\
\hline -r0 [rep0] & [rep0] is starting repetition. Default value: 0. \\
\hline & if $-\mathrm{v}$ is set, timing and some other info is printed. Default value: not set. \\
\hline & if - $\mathrm{g}$ is set, only the lowest energy solution is printed. Default value: not set. \\
\hline -sched [schedule] & $\begin{array}{l}\text { [schedule] specifies a schedule. It can either be lin, } \\
\text { exp or be a text file on the system which contains an } \\
\text { inverse temperature on every line. Default value: lin. }\end{array}$ \\
\hline [threads] & [threads] is the number of threads to run in parallel. Default value: OMP_NUM_THREADS. \\
\hline
\end{tabular}

Table 3: Command-line arguments of the provided algorithms.

\subsection{Running the codes}

Either of the previously described algorithms can be ran by using the appropriate executable, see table 2. Every algorithm follows the same command-line interface which allows one to specify the lattice using -1 , the schedule -sched, the number of sweeps $-s$ and the number of repetitions $-r$. Because repetitions are independent of each other, the codes can be trivially parallelised. The number of threads to run in parallel can be specified by -t. In cases where one uses one of the pre-programmed schedules, lin or exp, one can also specify the initial inverse temperature $\beta_{0}$ and the final inverse temperature $\beta_{1}$. In table 3 we summarise the full set of command-line arguments. A custom schedule can be loaded by using - sched followed by the name of a text file.

The input lattice files are plain text files with following structure: First line is the name of the lattice, and following $L+M$ lines contain $L$ couplings and $M$ local fields (not ordered). Each line contains three values $i, j$ and $c$. If $i=j$ the line specifies a local field on site $i$ of size $h_{i}=c$. Otherwise, the line denotes a coupling between spin $i$ and $j$ of value $J_{i j}=c$.

The output contains four columns: the energy, the number of times this energy is found, the success rate (the second column divided by the total number of repetitions), and the instance file name.

Sample input and output data are located in the example sub-directory of the source code distribution.

\section{Benchmarking}

We benchmark our codes on a 8-core Intel Sandy Bridge Xeon E5-2670 processor with hyper-threading enabled for Ising spin glass instances on the chimera graph. The codes are compiled by the GNU C++ compiler, version 4.7.2. To benchmark the codes, 1000 range-1 random 503-spin instances without fields are generated and annealing times are optimized as described in Sec. 3.2. The ground state energies of all instances were verified by exact solvers [17]. In multi-spin versions of the code, we run 64 repetitions simultaneously. Thus we have to round the number of repetitions to the nearest largest values that are multiples of 64 .

Generically, the multi-threaded version of the multi-spin code shows the best performance when the number of repetitions that is needed to find the ground state is large enough.

Times to find the solution with $99 \%$ probability, given by eq. 6, for the $99 \%$ quantile, see Sec. 3.2, are reported in tables 4, 5 and 6. Table 5 shows that we can reach 50 spin flips per nanosecond on a single Intel processor using our fast multi-spin and multi-threaded code. For the $99 \%$ quantile on a 503-chimera graph, the time to find the solution is less than $153 \mathrm{~ms}$ for range- 1 instances without random fields and less than $27 \mathrm{~ms}$ for instances with random fields.

Mean times to find the solution, given by eq. 7 , for the $99 \%$ quantile are reported in tables 7,8 and 9 . The mean time to find the solution is $8.2 \mathrm{~ms}$ and less than $40 \mathrm{~ms}$ for instances with and without fields for the fastest code. The time for instances with local random fields can be compared to the mean times to the solution reported for similar benchmarks on smaller problems in reference [5]. 


\begin{tabular}{l|r|r|r|r|r} 
Code & Sweeps & Repetitions & Init time in ms & Run time in ms & Spin flips per ns \\
\hline an_ms_r1_nf & 2000 & 7872 & 0.6 & 1190 & 6.65 \\
an_ms_r1_nf_v0 & 2000 & 7872 & 2.9 & 4172 & 1.90 \\
an_ss_ge_fi & 2000 & 7858 & 69.0 & 26206 & 0.30 \\
an_ss_ge_nf_bp & 1000 & 2301 & 19.4 & 6708 & 0.09 \\
an_ss_rn_fi & 2000 & 7858 & 0.7 & 25590 & 0.31
\end{tabular}

Table 4: Time $t_{T}$ to find the solution with probability 0.99 for the $99 \%$ quantile for range- 1 random instances without fields on the chimera graph of size 503 using 1 thread.

\begin{tabular}{l|r|r|r|r|r} 
Code & Sweeps & Repetitions & Init time in ms & Run time in ms & Spin flips per ns \\
\hline an_ms_r1_nf & 2000 & 7872 & 0.7 & 151.7 & 52.2 \\
an_ms_r1_nf_v0 & 2000 & 7872 & 139 & 491.1 & 16.1 \\
an_ss_ge_fi & 2000 & 7858 & 222 & 3261 & 2.42 \\
an_ss_ge_nf_bp & 1000 & 2301 & 58.0 & 876.6 & 0.66 \\
an_ss_rn_fi & 2000 & 7858 & 0.8 & 3177 & 2.49
\end{tabular}

Table 5: Time $t_{T}$ to find the solution with probability 0.99 for the $99 \%$ quantile for range- 1 random instances without fields on the chimera graph of size 503 using 16 threads.

\begin{tabular}{l|r|r|r|r|r} 
Code & Sweeps & Repetitions & Init time in ms & Run time in ms & Spin flips per ns \\
\hline an_ms_r1_fi & 1000 & 1792 & 0.8 & 25.4 & 35.5 \\
an_ss_ge_fi & 1000 & 1732 & 112 & 340.0 & 2.56 \\
an_ss_rn_fi & 1000 & 1732 & 0.8 & 335.6 & 2.59
\end{tabular}

Table 6: Time $t_{T}$ to find the solution with probability 0.99 for the $99 \%$ quantile for range- 1 random instances with fields on the chimera graph of size 503 using 16 threads.

\begin{tabular}{l|r|r|r|r|r} 
Code & Sweeps & Repetitions & Init time in ms & Run time in ms & Spin flips per ns \\
\hline an_ms_r1_nf & 2000 & 1728 & 0.6 & 261.2 & 6.65 \\
an_ms_r1_nf_v0 & 2000 & 1728 & 2.9 & 916.0 & 1.90 \\
an_ss_ge_fi & 2000 & 1667 & 68.7 & 5556 & 0.30 \\
an_ss_ge_nf_bp & 500 & 1000 & 10.8 & 1469 & 0.09 \\
an_ss_rn_fi & 2000 & 1667 & 0.7 & 5431 & 0.31
\end{tabular}

Table 7: Mean time $t_{M}$ to the solution for the $99 \%$ quantile for range-1 random instances without fields on the chimera graph of size 503 using 1 thread.

\begin{tabular}{l|r|r|r|r|r} 
Code & Sweeps & Repetitions & Init time in ms & Run time in ms & Spin flips per ns \\
\hline an_ms_r1_nf & 2000 & 1728 & 0.7 & 38.4 & 45.2 \\
an_ms_r1_nf_v0 & 2000 & 1728 & 139 & 122.9 & 14.1 \\
an_ss_ge_fi & 2000 & 1667 & 224 & 696.8 & 2.40 \\
an_ss_ge_nf_bp & 500 & 1000 & 30.0 & 189.8 & 0.66 \\
an_ss_rn_fi & 2000 & 1667 & 0.8 & 678.9 & 2.47
\end{tabular}

Table 8: Mean time $t_{M}$ to the solution for the $99 \%$ quantile for range-1 random instances without fields on the chimera graph of size 503 using 16 threads.

\begin{tabular}{l|r|r|r|r|r} 
Code & Sweeps & Repetitions & Init time in ms & Run time in ms & Spin flips per ns \\
\hline an_ms_r1_fi & 2000 & 384 & 0.7 & 7.5 & 25.7 \\
an_ss_ge_fi & 2000 & 377 & 111 & 75.4 & 2.51 \\
an_ss_rn_fi & 2000 & 377 & 0.8 & 74.5 & 2.54
\end{tabular}

Table 9: Mean time $t_{M}$ to the solution for the $99 \%$ quantile for range-1 random instances with fields on the chimera graph of size 503 using 16 threads. 


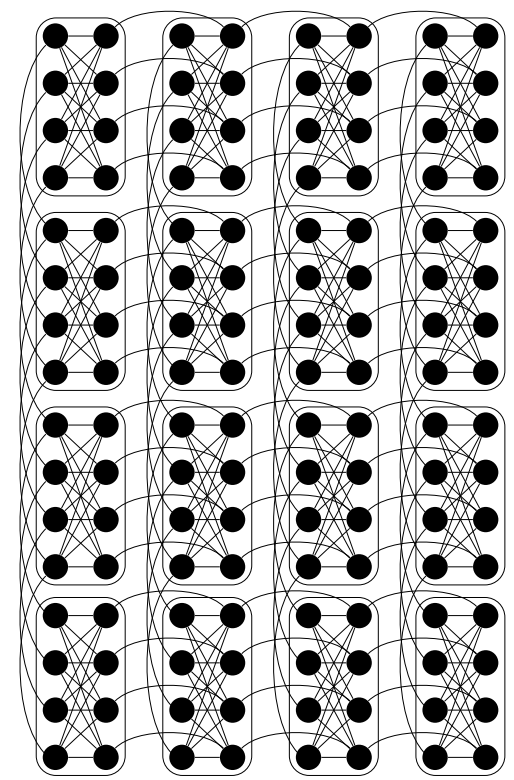

Figure A.3: $4 \times 4 K_{4,4}$ chimera graph having a total of 128 vertices.

\section{Acknowledgements}

This work was supported by the Swiss National Competence Center in Research on Quantum Science and Technology NCCR-QSIT and by the European Research Council through the ERC grant SIMCOFE. We acknowledge discussions with I. Pizorn, L. Gamper and C. McGeoch. M.T. acknowledges hospitality of the Aspen Center for Physics, supported by NSF grant PHY-1066293.

\section{Appendix A. The chimera graph}

Chimera graphs are lattices with bipartite fully connected unit cells consisting of $2 c$ vertices (denoted by $K_{c, c}$ ) distributed on a $L \times L$ grid - see figure A.3. D-Wave Two implements a transverse field Ising model on an $8 \times 8$ $K_{4,4}$ graph where every node represents a spin and every edge represents a coupling. We have used this graph for benchmarking our codes. In order to allow direct comparisons to an actual D-Wave device we use chimera graphs with missing vertices (due to fabrication issues) as on the D-Wave Two device located at the University of Southern California. Example input files using the specific 126 and 503 spin graphs used here are included in the software package.

\section{References}

[1] S. Kirkpatrick, C. D. Gelatt, M. P. Vecchi, Optimization by simulated annealing, Science 220 (4598) (1983) 671-680. doi:10.1126/ science.220.4598.671.

URL http://www.sciencemag.org/content/220/4598/671.abstract

[2] F. Barahona, On the computational complexity of Ising spin glass models, Journal of Physics A: Mathematical and General 15 (10) (1982) 3241 .

URL http: //stacks. iop.org/0305-4470/15/i=10/a=028

[3] H. Neven, G. Rose, W. G. Macready, Image recognition with an adiabatic quantum computer i. mapping to quadratic unconstrained binary optimization (2008), arXiv:arXiv:0804.4457.

[4] V. S. Denchev, N. Ding, S. V. N. Vishwanathan, H. Neven, Robust classification with adiabatic quantum optimization (2012). arXiv : arXiv : 1205.1148.

[5] C. C. McGeoch, C. Wang, Experimental evaluation of an adiabatic quantum system for combinatorial optimization, in: Proceedings of the 2013 ACM Conference on Computing Frontiers, 2013 
[6] R. H. Swendsen, J.-S. Wang, Replica monte carlo simulation of spin-glasses, Phys. Rev. Lett. 57 (1986) 2607-2609. doi:10.1103/ PhysRevLett.57.2607.

URL http://link .aps .org/doi/10.1103/PhysRevLett.57.2607

[7] C. J. Geyer, Markov chain monte carlo maximum likelihood, in: E. M. Keramidas (Ed.), Computing Science and Statistics: 23rd Symposium on the Interface, Interface Foundation, Fairfax Station, 1991, 1991, pp. 156-163.

[8] F. Romá, S. Risau-Gusman, A. Ramirez-Pastor, F. Nieto, E. Vogel, The ground state energy of the edwardsanderson spin glass model with a parallel tempering monte carlo algorithm, Physica A: Statistical Mechanics and its Applications 388 (14) (2009) 2821 - 2838. doi: $10.1016 / j$.physa.2009.03.036.

URL http://www.sciencedirect.com/science/article/pii/S0378437109002544

[9] J. J. Moreno, H. G. Katzgraber, A. K. Hartmann, Finding low-temperature states with parallel tempering, simulated annealing and simple monte carlo, International Journal of Modern Physics C 14 (03) (2003) 285-302. doi :10.1142/S0129183103004498.

URL http: //www . worldscientific.com/doi/abs/10.1142/S0129183103004498

[10] B. Block, P. Virnau, T. Preis, Multi-gpu accelerated multi-spin monte carlo simulations of the 2d ising model, Computer Physics Communications 181 (9) (2010) 1549 - 1556. doi :10.1016/j.cpc.2010.05.005.

[11] G. Bhanot, D. Duke, R. Salvador, A fast algorithm for the cyber 205 to simulate the 3d ising model, Journal of Statistical Physics 44 (5-6) (1986) 985-1002. doi:10.1007/BF01011918. URL http://dx.doi.org/10.1007/BF01011918

[12] N. Ito, Y. Kanada, Monte carlo simulation of the ising model and random number generation on the vector processor (1990) 753-763doi : 10.1109/SUPERC. 1990.130097.

[13] H. Rieger, Fast vectorized algorithm for the monte carlo simulation of the random field ising model, Journal of Statistical Physics 70 (3-4) (1993) 1063-1073. doi:10.1007/BF01053609. URL http://dx.doi.org/10.1007/BF01053609

[14] H.-O. Heuer, A fast vectorized fortran 77 program for the monte carlo simulation of the three-dimensional ising system, Computer Physics Communications 59 (2) (1990) 387 - 398. doi : 10.1016/0010-4655(90)90186-5. URL http://www.sciencedirect.com/science/article/pii/0010465590901865

[15] L. Pierre, T. Giamarchi, H. J. Schulz, A new random-number generator for multispin monte carlo algorithms, Journal of Statistical Physics 48 (1987) 135-149, 10.1007/BF01010404. URL http://dx.doi.org/10.1007/BF01010404

[16] T. Fischer, H. Lüthi, W. Petersen, A New Optimization Technique for Artificial Neural Networks Used in Prediction of Force Constants of Large Molecules, IPS research report, IPS, Interdisciplinary Project Center for Supercomputing, ETH-Zentrum, 1994. URL http: //books.google.ch/books?id=d-onPwAACAAJ

[17] T. F. Rønnow, Z. Wang, J. Job, S. Boixo, S. V. Isakov, D. Wecker, J. M. Martinis, D. A. Lidar, M. Troyer, Defining and detecting quantum speedup, Science 345 (6195) (2014) 420-424. doi : 10.1126/science.1252319. URL http: //www.sciencemag.org/content/345/6195/420. abstract 